\title{
La force et l'élégance. Les litotes dans la Passion bretonne de 1530
}

Strength and elegance: litotes in the 1530 Breton Passion

\section{Yves Le Berre}

\section{(2) OpenEdition}

1 Journals

Édition électronique

URL : https://journals.openedition.org//bl/1982

DOl : 10.4000/lbl.1982

ISSN : 2727-9383

Éditeur

Université de Bretagne Occidentale - UBO

\section{Édition imprimée}

Date de publication : 1 novembre 2011

Pagination : 123-149

ISBN : 978-2-901737-92-6

ISSN : $1270-2412$

\section{Référence électronique}

Yves Le Berre, «La force et l'élégance. Les litotes dans la Passion bretonne de 1530 », La Bretagne Linguistique [En ligne], 16 | 2011, mis en ligne le 01 mai 2021, consulté le 21 mai 2021. URL : http:// journals.openedition.org//bl/1982 ; DOl : https://doi.org/10.4000/lbl.1982

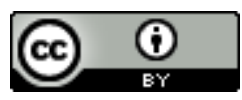

La Bretagne Linguistique est mise à disposition selon les termes de la Licence Creative Commons Attribution 4.0 International. 


\title{
La force et l'élégance. Les litotes dans la Passion bretonne de 1530
}

\begin{abstract}
$\mathrm{P}$ our Littré la litote est une «figure de rhétorique consistant à se servir d'une expression qui dit moins pour faire entendre plus». Et il cite l'exemple de Chimène, disant au Cid : «Va, je ne te hais point» pour lui signifier qu'elle l'aime, quoiqu'il vienne de tuer Don Diègue, son père. Pour Robert, c'est une «figure de rhétorique qui consiste à atténuer l'expression de sa pensée pour faire entendre le plus en disant le moins ». Il donne comme exemple le même demi-alexandrin de Corneille. Le Dictionnaire de linguistique de Dubois et alia n'en dit pas plus. Si l'on se fie à ces seules sources lexicographiques, on a le sentiment que la litote est une figure assez rare relevant de la rhétorique littéraire.

En réalité l'usage de la litote est extrêmement vivant et particulièrement répandu dans notre oralité quotidienne. Le Robert cite tout de même deux de ces litotes non littéraires : «ce n'est pas mauvais», «c'est pas dégueulasse», pour «c'est très bon». Écoutonsnous un peu parler et nous collectionnerons sans peine des centaines de litotes familières, parfois très familières : «c'est pas un con» 'il est très intelligent', «sans blague!» 'je parle tout à fait sérieuse-
\end{abstract}

\footnotetext{
* Professeur émérite de celtique, CRBC, UBO/ueb
} 
ment', «ç'est pas une lumière!» 'il est vraiment bête', «il s'prend pas pour une merde» 'il se croit un génie', «c'est pas la joie» 'c'est bien triste', «ça tourne pas rond» 'je me sens très mal', «c'est pas la mer à boire» 'c'est très facile', «c'est pas sa fête» 'il passe un mauvais moment', "ça va pas être triste» 'cela promet d'être terrible' etc. etc. La présence d'éléments scatologiques et l'ellipse constante de la négation devant le verbe situent ces expressions sur un barreau assez élevé de l'échelle de la parité.

D'autres litotes usuelles se rencontrent aussi en grande quantité à des niveaux de langue plus soutenus : "elle n'a plus toute sa tête» pour 'elle est complètement gâteuse', «il n'est sans doute pas très expérimenté» pour 'il n'y connaît absolument rien'. Et cela même en situation très disparitaire. Je relève par exemple dans des instructions officielles destinées aux enseignants des collèges : «l'ignorance du passé n'est guère propice à l'exercice d'une liberté bien comprise» et «la culture scolaire n'est pas étrangère à ses ${ }^{1}$ préoccupations». Mais on conviendra que ces expressions, étroitement apparentées à l'euphémisme et relevant du politiquement correct, forment un groupe d'usage un peu à part. L'immense majorité des autres (les premières citées) sont les manifestations d'un réel foisonnement créatif, d'un grand jeu langagier dont il serait intéressant de savoir s'il existe au même degré et avec la même constance dans d'autres langues. L'understatement anglais lui est assurément apparenté. On entend en breton : «N'eo ked 'n hanter douz eur genaoueg» 'ce n'est pas la moitié d'un imbécile', soit 'il est drôlement malin'.

Quoique tous les exemples cités jusqu'à présent soient à la forme négative, la négation n'est pas un constituant obligatoire de la litote. Si l'on demande à un père comment son fils joue au football et qu'il répond «il se défend»; si on lui demande encore «ta fille fait-elle des progrès au piano?» et qu'il répond «elle se débrouille», on comprendra que le fils est un dieu du stade et la fille une future Marguerite Long. C'est même, me semble-t-il, ce type de litotes qui répond le mieux à la définition courante «qui dit moins pour faire entendre plus ».

1. Il s'agit de l'élève de collège. 
J'ai entrepris il y a deux ans de refaire la transcription et la traduction des cinq textes du premier volume jamais imprimé en breton, en 1530, qui contient une Passion, une Résurrection et trois poèmes spirituels : Tremenuan an Ytron Guerches Maria, Pemzec leuenez Maria et Buhez Mabden. Au cours de ce travail je me suis heurté à des difficultés de compréhension provoquées par des séries d'expressions souvent récurrentes. Mes prédécesseurs, Hersart de La Villemarqué, Joseph Loth, Émile Ernault et Roparz Hemon, les ont généralement considérées comme des formules presque vides de sens que les auteurs de ces textes utilisaient un peu au hasard comme «chevilles» leur permettant de bâtir des vers réguliers. J'ai eu l'idée de regrouper ces expressions pour essayer d'y trouver des similarités et des oppositions qui m'aideraient à préciser éventuellement leur sens. Un grand nombre d'entre elles se sont trouvées relever de la catégorie rhétorique de la litote.

Ce qu'elles ont en commun est d'utiliser la négation du contraire plutôt que l'affirmation simple. Je trouve par exemple dans le volume de 1530 les couples suivants :

- Na lagaff tam (Buhez Mabden 5958) 'je ne mens nullement' vs Guiryonez a compsez rez splann (Passion 210) 'tu dis l'entière vérité';

- nen nachaf quet (Passion 81) 'je ne le nie point' vs Me lauar deoch flam ma mam quer (Passion 780) 'Je vous affirme, ma chère mère';

- Na doutet quet (Passion 321) 'n'en doutez pas' vs Credet dif (Passion 52) 'croyez-moi'.

J'ai d'abord effectué, non sans peine, un inventaire aussi complet que possible des litotes émaillant les 6104 vers du corpus. Mais d'une part je suis à peu près sûr de n'avoir pas effectué un travail vraiment exhaustif, d'autre part il n'est pas impossible qu'on puisse contester à certaines expressions retenues dans l'inventaire la qualité de litote.

En effet de nombreuses litotes sont si courantes qu'on ne les identifie plus comme telles qu'après réflexion : hep sy 'sans défaut', soit 'assurément', par exemple. Quelques autres, construites autour d'un verbe ou d'un verbe-nom, ne se comprennent que comme 
métaphores relevant de la langue mondaine : hep goulenn quen 'sans en demander davantage' qualifiant le verbe «obéir» pour signifier 'ponctuellement', hep sellet (poan) 'sans considérer (la peine)', pour qualifier un acte exécuté 'avec bienveillance'. D'autres encore sont à la limite de l'antiphrase ou de l'euphémisme, ou encore de l'hyperbole, et il est difficile de décider de leur identité. Lorsque dans la Passion Judas s'avance sur la scène pour nous dire sa honte d'avoir livré Jésus au Sanhédrin, il ne cherche pas à nous tromper sur sa véritable nature : Byzcoaz ne voe den ma quen drouc (Passion 1779) 'il n'y eut jamais homme aussi méchant que moi', soit 'je suis le plus méchant des hommes'; cet aveu est assurément une hyperbole (d'autres traîtres de l'histoire, ne lui en déplaise, doivent bien le dépasser en malignité), mais le fait qu'il soit exprimé de façon négative suffit-il à en faire également une litote? Il me semble que c'est le cas, mais je conçois qu'on puisse en douter.

Pour réaliser cet inventaire je me suis servi du dictionnaire alphabétique des mots des cinq textes, dictionnaire que j'avais déjà composé tout en travaillant à leur traduction ${ }^{2}$. J'en ai extrait tous les hep 'sans' (négation du nom) et les ne et na (négations du verbe) avec leurs contextes. J'ai éliminé les simples négations sans intention rhétorique ${ }^{3}$, ne gardant que les items qui me paraissaient en manifester une. Tel qu'il est constitué aujourd'hui, cet inventaire contient 548 litotes. Une litote apparait donc tous les 11 vers en moyenne dans l'ensemble du corpus, ce qui suffit largement à en faire un véritable phénomène stylistique et rhétorique digne d'être étudié pour lui-même.

J'ai ensuite analysé cette collection selon différents critères : la forme que revêtent les différentes expressions et la façon dont elles s'insèrent dans le flux syntaxique, la manière dont elles «font litote» et leur contenu de sens; leur distribution entre catégories et entre les cinq textes; leurs caractères sociolinguistiques et leur visée énonciative. J'essaie enfin de faire en conclusion la synthèse des enseignements glanés tout au long de l'analyse.

2. En partie grâce au logiciel d'indexation Kwic-Magic.

3. Rac nen quirif muy bizhuyquen (v. 2388) 'car je ne l'aimerai plus jamais' dit la femme de Pilate parlant de son époux. 


\section{Syntaxe de la litote}

Accidents volontairement provoqués dans le déroulement du discours, un peu comme une syncope ou un contretemps dans un flux rythmique régulier, les litotes peuvent revêtir plusieurs aspects selon la nature du segment de syntaxe sur lequel elles sont destinées à attirer l'attention.

\section{La litote développée en phrase ou en proposition relative}

Une centaine de litotes contiennent en elles-mêmes l'élément sémantique qu'elles mettent en relief :

Pendant le repas chez Simon, Marthe dit à son frère Lazare : Nouz guellaff plean ouz louenhat 'je ne vous vois pas pleinement vous réjouir', soit 'vous avez l'air tout triste' (Passion 124);

Tout en marchant vers Emmaüs, Cléophas et Luc parlent de Jésus : Nedoa nygun dezan vnuan 'Aucun n'était son égal', soit 'il les surpassait tous' (Résurrection 4159);

Le Christ annonce à sa mère les moments difficiles qu'elle devra traverser avant d'être couronnée aux cieux: Hoguen tribuill, ma ytron quer, a vezo ne vezo dister 'Toutefois, ma chère Dame, il y aura des épreuves qui ne seront pas de peu d'importance', soit 'qui seront terribles' (Tremenuan 4960-4961);

Jésus rassasie cinq mille personnes avec cinq pains seulement : Nemet dou pesq ne cresquas quen 'Si ce n'est deux poissons, il ne multiplia pas autre chose', soit 'en tout et pour tout' (Pemzec Leuenez 5705);

Fût-il un grand de ce monde, l'homme qui porte le plus léger fardeau de péché nedeu quet nepret dipreder 'n'est certes jamais sans souci', soit 'est toujours en grand danger (de damnation)' (Buhez Maden, 5931).

\section{La litote proposition incise}

Quand elle se présente sous la forme d'une proposition complète et autonome, la litote flanque un autre segment de phrase qu'elle met en relief, en quelque sorte par surlignage :

Judas médite de trahir son maître : Hac en rento, ne vezo quen, dezo seder 'et je le leur livrerai sans faute, il n'en sera pas autrement', soit 'j'y suis bien déterminé' (Passion 299-300); 
Marie rend grâces à son fils d'avoir racheté l'humanité, désormais frealset net ne fel netra 'tout entière libérée, il ne s'en faut de rien', soit 'parfaitement' (Résurrection 3684)

Aux noces de Cana, hennez nendeu queffrin, an map man dre amour [a] dour a guere guin 'cet enfant, cela n'est pas secret, fit d'eau du vin, par amour', soit 'changea l'eau en vin, l'histoire est bien connue' (Tremenuan 5463);

Le jour du Jugement, Dieu descendra sur terre accompagné d'anges jnnumerabl nedynt fablou 'innombrables, ce ne sont pas fables', soit 'littéralement innombrables' (Buhez Mabden 6011).

\section{La litote extension du groupe nominal}

Toujours introduites par la préposition hep 'sans' suivie d'un nom ou d'un verbe-nom, elles ont une fonction qualificative et apparaissent comme des variantes morphologiques des adjectifs préfixés en di- (dinam, dynoas, dans les exemples ci-dessous).

Reprochant aux Juifs leur ingratitude envers Jésus, Marie rappelle les services a gueureu douz re hep merit 'qu'il rendit à des gens sans mérite' soit 'qui en étaient indignes' (Passion 2657);

Jésus, ressuscité dans toute sa gloire, rappelle que enouf hep muy ezedi an squient 'en moi sans plus réside la connaissance', soit 'en moi seul' (Résurrection 3570);

Peu avant qu'elle ne meure, Gabriel vient rendre visite dan guerches hep si maria 'à la vierge sans défaut, Marie', soit 'immaculée' (Tremenuan 4987);

Le pécheur s'adresse lui aussi à la guerches dinam hep tam pechet 'vierge sans tache et sans aucun péché' (Pemzec Leuenez 5640). La litote est ici redondante.

Dieu maudit le méchant: Eat oas dynoas hep quen lastez 'tu étais parti innocent, sans autre souillure' (Buhez Mabden 6061). La litote est ici aussi redondante.

\section{Morphologie de la litote}

Quand elle est développée en phrase ou en proposition relative, la litote relève simplement de la syntaxe de la phrase négative.

Lorsqu'elle apparaît sous la forme d'une proposition incise ou d'une extension du groupe nominal, la litote s'annonce par deux 
entrées différentes : soit les deux négations du verbe (ne et $n a$ ), soit la négation du nom (la préposition hep).

\section{Ne ou na + verbe (+COD)}

Judas, dit le démon, tu n'as plus rien à faire sur la terre; après ta trahison, nez priso den 'Nul ne te prisera', soit 'tous te haïront' (Passion 1250);

Quand adviendra le dernier jour, chacun sera jugé selon ses mérites: Me ho cafo vndro ne mano penn 'Je les retrouverai tous, il ne demeurera personne', soit 'tous sans exception seront présents' (Résurrection 3603);

A qui célébrera sincèrement la fête de l'Assomption, An roe hep mar quet ne falho 'Le Seigneur ne fera certes pas défaut' soit 'sera secourable' (Tremenuan 5593);

Les avaricieux se croient éternels, mais ho vost ho stat pell ne pado 'leur vanité, leur gloire, ça ne durera pas longtemps', soit 'seront éphémères' (Buhez Mabden 5841).

\section{Ne ou na + copule (+ attribut)}

Le diable a visité l'épouse de Pilate pendant son sommeil: Guelet emeux ha nendeux sy 'j'ai vu, et il n'y a pas de défaut...', soit 'incontestablement' (Passion 2378) ;

La Madeleine pleure son maestr doucc ha huec nendeu quen 'maître doux et aimable, qui n'est plus', soit 'qui est mort' (Résurrection 3780 ) ;

Marie rappelle à Jean que Jésus 1'a confiée à lui : Ha ne voe hep mar diuar goap 'et ce ne fut certes pas par plaisanterie', soit 'impérativement' (Tremenuan 5169);

Même si les puissants ont de la peine à admettre qu'ils perdront tout en mourant, na lagaff tam cleu flam aman 'je ne mens nullement, écoute-moi bien', soit 'c’est la pure vérité' (Buhez Mabden 5958).

\section{Hep + nom}

Jésus explique à sa mère qu'il doit mourir : So dif ret hep quet contredit 'cela m'est sans nul contredit nécessaire', soit 'inéluctablement' (Passion 408) ;

Nicodème demande à Joseph d'Arimathie d'expliquer comment 
Jésus l'a délivré de prison; Joseph accepte de le faire hep nep finction, autronez 'sans nulle dissimulation, Messeigneurs', soit 'très franchement' (Résurrection 4680);

Lorsque Gabriel vient lui annoncer sa mort proche, Mary hep gront a respontas 'Marie répondit sans protestation', soit 'bénignement'(Tremenuan 5112);

Marie a porté l'enfant Jésus dans ses entrailles nau miz hep difforch 'neuf mois sans séparation', soit 'constamment pendant neuf mois' (Pemzec Leuenez 5658) ;

Lorsque nous mourrons, puissions-nous obtenir an gracc man hep huanat 'cette grâce sans soupir', soit 'pleine et entière' (Buhez Mabden 6094).

\section{Hep + verbe-nom (+COD)}

Bourrelé de remords, Judas veut restituer les trente deniers dan princet hep quet arretaf 'aux pontifes, sans s'attarder du tout' ou 'sans hésiter', soit 'sur-le-champ' ou 'résolument' (Passion 1748);

Accusé de lèse-Sanhédrin, Joseph d'Arimathie croupit trois jours au fond d'une oubliette, hep guelet gleur 'sans voir lueur', soit 'dans une complète obscurité' (Résurrection 4700) ;

Juste avant la mort de Marie, Pierre prêchait à Antioche lorsqu'il fut miraculeusement transporté à Jérusalem : ez duiz aman hep dale tam 'j'arrivai ici sans nullement tarder ', soit 'subitement' (Tremenuan 5058);

Le pécheur prie Marie d'intercéder auprès de son fils afin $e z$ pardonno hep quet fellell 'qu'il lui pardonne, sans nullement y manquer', soit 'à coup sûr' (Pemzec Leuenez 5676);

Nous, les hommes, nous livrons au péché hep sellet quen 'sans y regarder autrement', soit 'à la légère' ou 'volontiers' (Buhez Mabden 5882).

\section{Hep + copule + attribut}

Jésus envoie Jean et Pierre préparer la Pâque; quand ils seront en présence du patron de l'auberge, lyuyrit scaff hep bezaff ven 'dites(-lui) vivement, sans être vains', soit 'succinctement' (Passion 956);

Si Jésus ne se sacrifie pas, l'humanité demeurera hep bezaf quet 
frealset tam 'sans être du tout soulagée le moindrement', soit 'dans les souffrances les plus terribles' (Résurrection 3518).

\section{Les extensions}

Ces bases récurrentes hep + nom ou verbe-nom et $n a, n e+$ verbe ou copule peuvent recevoir des extensions variées qui en amplifient le caractère affirmatif. Ces extensions peuvent même être associées dans la même expression.

- quet placé à gauche du nom ou du verbe-nom : hep quet sy 'sans nul défaut', soit 'assurément'; hep quet mar 'sans aucun doute'; hep quet fellell 'sans nullement faillir', soit 'à coup sûr'; hep quet chom 'sans demeurer du tout', soit 'sur-le-champ';

- quet placé à droite du nom ou du verbe-nom : hep dout quet 'sans doute aucun'; hep nech quet 'sans contrariété aucune', soit 'bénignement'; hep dale quet 'sans tarder un instant'; hep lacat quet contredy 'sans formuler la moindre objection', soit 'de très bon gré';

- quet a (partitif) placé à gauche du nom : hep quet a abec 'sans aucune raison', hep quet a reuil 'sans nulle joie';

- quen toujours placé à gauche d'un nom : hep quen mez 'sans autre objection', soit 'assurément'; hep quen lastez 'sans autre souillure', soit 'immaculé';

- quen placé à droite d'un pronom : hep netra quen 'sans rien d'autre', soit 'en tout et pour tout';

- quen toujours placé à droite d'un verbe-nom : hep sellet quen 'sans y regarder autrement', soit 'avec bienveillance'; hep sezlou quen 'sans écouter autre chose', soit 'en se hâtant';

- nep placé à gauche d'un nom : hep nep goap 'sans aucune plaisanterie', soit 'assurément'; hep nep blam 'sans aucun défaut', soit 'immaculé';

- tam, tro, pret, vn pret, pas, affet, a se ou muy placés à droite d'un nom ou d'un verbe-nom : hep amouc muy 'sans plus de retard', soit 'sur-le-champ', hep tardaf pret 'sans tarder un instant', soit 'immédiatement';

- affet, dre affet, apret ou en bet placé à droite d'un nom ou d'un pronom : Gant ioae hep abaff dre affet 'avec une joie en vérité sans embarras', soit 'l'air tout réjoui'; Hep goap apret 'sans aucunement plaisanter', soit 'très sérieusement'; 
- tam placé à gauche ou à droite d'un nom : hep tam pechet 'sans une once de péché', soit 'immaculé', hep nech tam 'sans la moindre contrariété', soit 'bénignement';

- un qualificatif placé à droite du nom : hep guer gou 'sans mot mensonger', soit 'véritablement'; hep buez glan 'tout à fait sans vie', soit 'raide mort';

- une seconde litote enchaînée : hep mar na sy 'sans doute ni défaut', soit 'très certainement'.

\section{Les deux modalités de la litote}

En y regardant de plus près, le corpus des litotes n'est pas aussi homogène qu'il y paraît dans son fonctionnement. Deux modalités assez différentes du «faire litote» s'y distinguent.

\section{La négation simple}

Certaines d'entre elles procèdent par la simple négation d'une qualité axiologiquement neutre ou positive, un fait naturel ou une vertu, par exemple :

Si les apôtres savaient lequel d'entre eux doit trahir le Christ, $e z$ ve hep truez labezet 'il serait lapidé sans pitié' (Passion 1187);

Thomas s'adresse au Christ ressuscité : Huy so hep diuez na dezrou 'vous êtes sans fin ni commencement', soit 'vous êtes éternel' (Résurrection 4831);

Lorsque Thomas rejoint les autres apôtres après avoir vu la Vierge montant aux cieux, son visage exprime une joie nen doae berr quentel eguelet 'qu' on lui avait vue il n'y avait pas peu de temps', soit 'qu'on ne lui avait pas vue depuis longtemps' (Tremenuan 5309);

Le récitant annonce qu'il va énoncer la septième joie de Marie : An seizuet teuell quet nem deur 'il ne me convient point de taire la septième', soit 'je veux réciter la septième' (Pemzec Leuenez 5687);

La mère de Dieu est da pep hep span glan roanes 'de chacun sans cesse sainte reine', soit 'sainte reine des hommes pour l'éternité' (Buhez Mabden 6008).

\section{La négation double}

Les autres procèdent par la négation d'une qualité axiologiquement négative, un défaut, un manque ou un vice, par exemple : 
La prophétie annonçant le sacrifice de Jésus est scryuet hep gou en lefrou bras 'écrite sans mensonge dans de grands livres', soit 'fidèlement consignée dans des livres vénérables' (Passion 579);

Le Christ ressuscité s'adresse à Joseph : nendouf pas fantasy 'je ne suis pas une illusion', soit 'je suis bien réel' (Résurrection 3701);

Jésus annonce à sa mère qu'elle montera aux cieux hac eno ez vech hep nep mez 'vous y serez sans nulle peine', soit 'vous y demeurerez quiètement' (Tremenuan 4908);

Lorsque tu seras damné, a huen eno nez vezo mez 'étendu sur le dos, alors, tu n'auras plus d'objection', soit 'alors, gisant, tu seras impuissant' (Buhez Mabden 5889).

$\mathrm{Au}$ lieu d'exprimer directement la qualité : ha so bizhuyquen da menell (v. 1144) 'qui doit demeurer éternellement', an bresel calet meurbet fier (v. 697) 'la guerre rude, fort cruelle', maru gant riou (v. 1580) 'mort de froid', ces litotes nous font passer par la négation du contraire. Ce faisant, elles confèrent à l'expression une énergie supplémentaire : troublant un instant l'esprit et l'obligeant à «retourner» l'expression pour la comprendre, elles placent la qualification sous une lumière plus vive et lui donnent une certaine emphase ${ }^{4}$.

\section{Sémantique et sémiologie}

Même si en principe n'importe quel élément du discours peut être exprimé sous la forme d'une litote, le classement des items du corpus fait apparaître de grandes différences entre des thèmes particulièrement perméables à la litote et d'autres qui le sont beaucoup moins. Je propose ci-dessous une tentative de classement regroupant par séries les litotes portant sur le même thème. Chaque série s'ouvre par un mot ou une expression résumant ce thème; une traduction littérale permet d'estimer le degré d'idiomatisme ou de métaphorisation de chaque litote.

4. Elles troublent l'esprit parfois plus qu'un instant. En témoigne la faute récurrente en français: «Vous n'êtes pas sans ignorer...» (négation triple), pour "vous n'êtes pas sans savoir» (négation double). 


\section{L'appel insistant à la confiance en la vérité énoncée}

Affirmer : NE [NACH-] 'ne pas nier'.

Assurément: HEP ABAFF 'sans embarras'; HEP ABUS 'sans tromperie'; HEP AUIS 'sans illusion'; HEP BEZAF BREF 'sans être bref?'; HEP CHOM 'sans demeurer'; HEP COMPS GOU 'sans dire de mensonge'; HEP CONTREDY 'sans contredit'; HEP DOUT 'sans doute'; HEP ENOE 'sans ennui'; HEP EXCES 'sans faute'; HEP FAE 'sans faille'; HEP FAUT 'sans faute'; HEP FAZIAFF 'sans faire d'erreur'; HEP FELLELL 'sans manquer'; HEP FINCTION 'sans mensonge'; HEP GAES 'sans moquerie'; HEP GOALL 'sans faute'; HEP GOAP 'sans plaisanterie'; HEP GOU 'sans mentir'; HEP GUER BREF 'sans mot bref ?'; HEP LACAT SY 'sans admettre de défaut'; HEP MAR 'sans doute'; HEP MEZ 'sans objection'; HEP NECH 'sans gêne'; HEP NOAS 'sans querelle'; HEP OBER RUS 'sans faire de plaisanterie'; HEP QUEN SON 'sans autre souci'; HEP QUEN TRA 'sans autre chose'; HEP SAFAR 'sans conteste?'; HEP SY 'sans défaut'; HEP TRETE 'sans négociation'; HEP TRIG 'sans tromperie'; NEDEU BOURT 'ce n'est pas mensonge'; NEDEUX DOUT 'il n'y a pas de doute'; NEDEU GOU 'ce n'est pas mensonge'; NEDEU QUEN 'il n'en va pas autrement'; NEDEUX GOAT 'ce n'est pas d'objection';

NEDEUX MAR 'il n'y a pas de doute'; NENDEU GOAP 'ce n'est pas plaisanterie'; NENDEUX SY 'il n'y a pas de défaut'; NEDOUF FLATER 'je ne suis pas menteur'; NEDYNT FABLOU 'ce ne sont pas fables'; NE [FALL-] 'ne pas manquer'; NE [GRA-] DOUT 'ne pas douter'; NE [GRA-] GOAP 'ne pas plaisanter'; NE [GRA-] LE 'ne pas faire offense?'; NE [GRA-] QUEN 'ne pas faire autre chose'; NE [GRA-] SY 'ne pas supposer un défaut'; NE [LAQU-] SY] 'ne pas admettre d'erreur'; NE VEZO MUY 'tu n'en auras pas davantage'. ${ }^{5}$ Nonnem doutomp tam autramant 'Nous n'avons aucun doute à ce sujet' = 'nous sommes péremptoires'; Na lagaff tam 'Je ne mens nullement' = 'je l'affirme;

Au complet : NE [FELL-] VNAN 'il n'en manque pas un'.

5. Je ne donne en capitales que les formules les plus stéréotypées; les autres sont notées en minuscules, ce qui permet de distinguer les deux grandes catégories de litotes relativement à la syntaxe. 
Au plus haut point : HEP PREZEC MUY 'sans en dire davantage'. Notoirement : NENDEU QUEFFRIN 'ce n'est pas secret'.

\section{Le rappel à la règle sociale ou morale}

Inconsidérément : HEP EZOM 'sans nécessité'; HEP MECHER 'sans besoin'; HEP RET 'sans nécessité'.

Indigne(ment) : HEP GONIT 'sans bénéfice'; HEP MERIT 'sans mérite'.

Indûment: HEP ABEC 'sans cause'; HEP DELLIT 'sans mérite'; HEP UTILITE 'sans utilité'

ne falhe ez ve quet dissimulet 'il ne faudrait pas que cela soit celé' $=$ 'tous doivent le savoir'; Rac ne ve seant nep andret 'Il ne serait nullement séant'; ne ve quet raeson 'il ne serait pas juste'; Ne ve quet se enor deoch 'Il ne serait pas honorable pour vous'; Hennez ne ve quet competant 'Il ne serait pas convenable'; Ne ve quet raeson consonant ' $\mathrm{Ce}$ ne serait pas raison convenable'; ne allhe ez ve quen 'il ne pourrait en être autrement' = 'c'était nécessaire'; Ne falhe quet ez grahet quen 'Il n'eût pas fallu que vous agissiez autrement'; Hennez ne apparchant nep andret 'Il ne t'appartient pas de'; Ne ve quet raeson deboner 'Il n'y aurait aucune bonne raison'; rac nedoa seant 'car ce n'était pas convenable'; na fallo quet 'qu'il n'y manque pas'; Gourchemenn doe ne delchsot quet 'vous n'avez pas observé le commandement'; Ha ne voe hep mar diuar goap 'Ce ne fut certes pas pour plaisanter'.

\section{Le refus poli}

Refuser : NE [TEUR-] 'il ne convient pas'; Nep e pep guys dre coantis na pris quet 'Ceux qui ne daignent pas'; En effet ma ne queritu 'Si vous ne voulez pas'; Pan nozeux difme autreet 'Vous n'avez pas octroyé'; Nozeux nep guys oboysset 'Vous n'avez aucunement accédé';

Ne reit diffme dre nep eur 'Vous ne m'accordez pas'; rac ne pris quet credet lauaret guer 'Il ne daigne pas s'exprimer'; Malchus a ne prisez te trauell 'ne daignes-tu pas'; Ma ne queret hep contredy 'Vous ne consentez pas'; Nendeu ma spy muy e cruciffiaf 'Mon intention n'est pas de le crucifier'. 


\section{L'expression d'un sentiment fort}

Désirer ardemment: NE [SONG-] QUEN 'ne pas penser à autre chose'.

Divers: Eguyt an boet nem em hetaf 'En ce qui concerne la nourriture, je ne me réjouis pas' = 'la nourriture me dégoûte'; Nouz guellaff plean ouz louenhat 'Je ne vous vois pas pleinement vous réjouir'= 'vous avez l'air tout triste'; Ha nep huy en goar no carquet 'Qui ne vous aiment pas'= 'qui vous haïssent'; Dalchet dren bro nez priso den 'Nul ne te prisera'= 'Tous te haïront'; An re nen care 'Ceux qui ne l'aimaient pas'= 'Ceux qui le détestaient'; Da contenancc ne auancc den 'Ton allure ne réconforte personne' = 'tu es horrible'; $\mathrm{Na}$ vech quet car Cesar ny en goar acc 'Vous ne seriez pas l'ami' = 'Vous seriez l'ennemi'; Nonobstant nedouf quet hetus 'Je n'en suis pas heureux' = 'cela me déplaît'; Ha bizhuyquen ne louenhaf 'Je ne me réjouirai plus jamais' = 'je vivrai dans un perpétuel chagrin'; $\mathrm{Na}$ ioa ne graf nen guelaf beu 'Je ne connaîtrai pas la joie' = 'je serai malheureux'; Nonn em cafemp quet en seder 'Nous n'étions pas gaillards' = 'nous étions paralysés'; Nendoann quet quen ferm dan termen 'Je n'ai pas été très ferme' = 'j' ai été lâche'; Na nedomp quet bezet enn andret sur 'Nous n'avons pas été assurés' = 'nous avons failli'; Rac bizhuyquen dre den ne louenhif 'Je ne me réjouirai plus jamais' = 'je vivrai dans un perpétuel chagrin'; Ha nedeux queuzet nemedouf 'Il n'y a que moi de chagriné' = 'je suis le plus malheureux des hommes'.

\section{L'expression d'un état ou d'une attitude}

Bénignement : HEP ALLAS 'sans plainte'; HEP ESTRENUA 'sans dureté'; HEP GRONT 'sans protestation'; HEP HUANAT 'sans soupir'; HEP REFFUS 'sans refus'; HEP SELLET 'sans y regarder'; HEP SELLET POAN 'sans considérer la peine'; HEP VILENY 'sans bassesse'.

Cruel(lement) : HEP DIFFRAE 'sans soulagement'; HEP ESPERN 'sans ménagement'; HEP FLAIG 'sans mollesse'; HEP QUERNEZ 'sans témoignage d'affection'; HEP SOULACC 'sans consolation'; HEP TRUEZ 'sans pitié'; Ez tourmanter na no esperner quet 'on ne les épargnera pas'. 
Effrontément : HEP DOEN PRIS 'sans considérer la valeur'; HEP OBER CAS 'sans faire cas'.

En harmonie : No deuoe nepret contredy 'Qui ne furent jamais en désaccord'.

Franchement : HEP DIGAREZ 'sans circonlocutions'; NE [COMS-] VAEN 'ne pas parler pour ne rien dire'.

Hardiment : HEP DOUGAF MES 'sans craindre la honte'; HEP DOUGANCC 'sans crainte'; HEP DOUGIAF GOUR 'sans craindre personne'; HEP ESTON 'sans ébranlement'; HEP SPONT 'sans crainte'; Ne dougeas nygun a nep tu 'Il ne se soucia de personne'.

Heureux, heureusement : HEP POAN 'sans peine'.

Immaculé : HEP BLAM 'sans faute'; HEP CLEFUET 'sans faute'; HEP LASTEZ 'sans souillure'; HEP ORDUR 'sans saleté'; HEP PECHET 'sans péché'; HEP TACHENN ‘sans tache'; HEP TORFET 'sans forfait'.

Indemne : HEP GOA 'sans mal'; HEP TRAUELL 'sans torture'.

Indifférent: HEP BOUT BUANCEC 'sans être accablé'; HEP COMPASSION 'sans compassion'.

Innocent : HEP OBER BREIG 'sans avoir fait de tort'; HEP OBER DROUC 'sans faire de mal'; HEP OBER EXCES 'sans commettre de faute'; HEP PECHIFF 'sans pécher'; So merch ha mam ne voe blamet 'Qui ne fut en faute'.

Quiet, quiètement: HEP ANES 'sans malaise'; HEP BRUT 'sans bruit'; HEP ESMAE 'sans trouble'; Nep aoun nouz bezet, Noz bezet aoun quet ' $N$ 'ayez nulle crainte'.

Résolu(ment): HEP ARRETAF 'sans hésiter'; HEP CRETHAT 'sans retenue?'; HEP DEBAT 'sans contestation'; HEP DEBATAF 'sans contester'; HEP DECLINAFF 'sans se dérober'; HEP FLAIG 'sans mollesse'; HEP LACAT CONTREDY 'sans admettre de contestation'; HEP SEZLOU QUEN 'sans écouter autre chose'; HEP TERRIF 'sans contrevenir'; NE [MENN-] QUEN 'ne pas vouloir autre chose'.

Spontanément : HEP REQUETIF 'sans être requis'. 


\section{Autour du temps}

Brièvement : HEP BEZAFF VEN 'sans être vain'.

Comptant : HEP REIF TERMEN 'sans accorder de délai'.

Constamment: HEP DIFFORCH 'sans séparation'; HEP REPOS 'sans repos'; HEP SQUET 'sans cesse?'; Her dre beuif ne seczif dez 'Je ne cesserai pas un seul jour'.

Éternel : HEP DIUEZ NA DEZROU 'sans fin ni début'; Bizhuyquen finuez nouz bezo 'Vous n'aurez jamais de fin'.

Longtemps : Nen doae berr quentel eguelet 'Il n'y avait pas peu de temps'; Ho vost ho stat pell ne pado 'Ne dureront pas lontemps' = 'seront éphémères'.

Perpétuel(lement)

HEP DIANC 'sans échappatoire'.

HEP DYSTENN 'sans relâche';

HEP FINUEZ 'sans fin'.

HEP SPAN 'sans écart'.

Sur-le-champ : HEP AMOUC 'sans retard'; HEP DALE 'sans tarder'; HEP DIFFERAFF 'sans différer'; HEP EHANAFF 'sans prendre de repos'; HEP REMET 'sans remise'; HEP REMU 'sans délai'; HEP TARDAFF 'sans tarder'; NE [CHOM-] PELL 'ne pas demeurer longtemps'.

\section{L'expression emphatique d'une restriction}

Discrètement : HEP CAQUET 'sans bavardage'.

Dans l'obscurité : HEP GUELET GLEUR 'sans voir lueur'.

D'une seule pièce : HEP GRY 'sans couture'.

Mort: HEP BUEZ 'sans vie'; NENDEU QUEN 'qui n'est plus'; Breman pa nac eu 'Il n'est plus'.

$\underline{\mathrm{Nu}}$ : HEP BARR DILLAT sans aucun vêtement.

Pour toute pitance : HEP QUEN REFECTION 'sans autre repas'.

Seulement: HEP MUY 'sans plus'; HEP QUEN 'sans autre'; An oll ma holl ioa nemoa muy 'Je n'en avais pas davantage' = 'il était mon unique joie'; Nep dif ayoa na nemoa muy 'Je n'en avais pas davantage' = 'je n'avais que lui'; Pelech ezout ha na ve quen 'Il 
n'y aurait pas autre chose' = 'cela seulement'; Nemet dou pesq ne cresquas quen 'Il ne multiplia pas autre chose' = 'en tout et pour tout'.

\section{L'expression emphatique d'un absolu}

Incroyable(ment) : NE [CRED-] DEN 'nul ne le croit'.

Inéluctable(ment) : HEP BOUT FREALSET 'sans être soulagé'; HEP BEZAF RECONCILIET 'sans être réconcilié'; HEP BOUT REDIMET 'sans être racheté'; HEP CAFOUT REPETANCC 'sans avoir de recours'; NE [GALL-] MUY (QUEN) 'n'en pouvoir mais?'.

Inerte : HEP NERZ 'sans énergie'.

Irrévocablement : HEP QUEMERET AZRECTET 'sans éprouver de repentir'; NE [FALL-] QUEN (MUY) 'ne pas falloir autrement'.

Semblable : HEP DIFFERANCC 'sans différence'.

Universellement : HEP CHENCHAFF DEN 'sans délaisser quiconque'; HEP EXCEPTAFF DEN 'sans excepter quiconque'; Me ho cafo vndro ne mano penn 'Il ne restera personne'; Nedoa nygun dezan vnuan 'Aucun ne l'égalait'; Ne guell nep quentel fellell tro 'Elle ne peut jamais faillir'; Nedeux a nep re a ve glan 'Il n'existe personne qui soit pur'.

Divers : Nendouf pas fantasy 'Je ne suis pas une illusion' = 'je suis bien réel'; $\mathrm{Na}$ oar nep tro nes vezo mez 'Tu n'auras jamais rien de fâcheux' = 'tu seras en parfaite sécurité'; A huen eno nez vezo mez 'Tu n'y pourras plus rien' = 'tu seras impuissant; Nedeu quet nepret dipreder 'Il n'est jamais sans souci' = 'il est toujours en grand danger'.

\section{L'expression emphatique d'une qualité}

HEP REUIL 'sans joie'; Nedeuz pez anezy dien 'Il n'y a rien d'intact en elle' = 'elle ne vaut plus rien'; Gant an mann han nerz hep terzyen 'la force sans fièvre?'; A vezo ne vezo dister 'Qui ne seront pas négligeables' = 'qui seront terribles'; Acc an gracc man hep huanat 'La grâce sans soupir'. 


\section{Divers}

Quet e techet ne gallaff tro 'Je ne puis l'éviter' = 'j'y suis résolu'; Gant an princet nedy quet cuyt 'Tu ne partiras pas libre' = 'tu auras affaire à eux'; Cofes a gris ne nachis quet 'Je n'ai pas nié' = j'ai tout avoué'; No deueux quet dellezet quen 'Ils n'ont pas mérité autre chose' = c'est bien fait pour eux'; Ha dre na grae brut 'Il ne disait mot' = 'il restait silencieux'; Nep stat ouz e coufhat na teu 'Qui ne sort nul instant de sa pensée' = 'qui l'obsède'; Ne leso quet da monet pell 'Ne te laissera pas aller loin' = 'te stoppera net'; Ne galsech quet ma muy hetaf

'Vous ne sauriez me réjouir davantage' = 'vous me comblez'; Ne fell quet bezaf non sauant 'Il ne faut pas être ignorant' = 'il faut absolument savoir'; Ez chomif na ne flachif quet 'Je ne bougerai pas' = 'je demeurerai'; Ned eu quet expres am deseu 'Cela n'est pas assuré' = 'je pense qu'au contraire...'; Ne quelhet netra ma credet 'Vous n'y perdrez rien' = 'vous serez grassement payée'; Na ne menna quet arretaf 'Je ne veux pas tarder' = 'je me hâte'; Bizhuyquen tristez nembez muy 'Je n'ai plus jamais de tristesse' = 'je suis au comble de la joie'; An roe hep mar quet ne falho 'Ne fera pas défaut' $=$ 'sera secourable'.

\section{La distribution}

J'ai relevé dans l'ensemble du corpus 252 litotes différentes, moyennant les réserves déjà exprimées sur l'exactitude de mon décompte. 191 de ces litotes, soit plus de $75 \%$, ne figurent qu'une seule fois dans les 6104 vers considérés. Les 61 autres y figurent de 2 à 50 fois chacune. Au total leur taux de récurrence moyen est à peine supérieur à $2(2,17)$. Quoique toutes les supposées «chevilles» dont la doxa fait l'une des plaies de la littérature bretonne de cette époque ne soient pas des litotes, ce nombre suffit déjà à montrer que l'immense majorité des litotes ne sauraient en aucun cas être considérées comme des chevilles à peu près vides de sens. La plus récurrente de toutes, hep sy 'assurément', ne figure elle-même que dans un vers sur cent vingt-deux. 


\section{La distribution entre séries formelles}

Ces différents groupes établis sur la base de la forme, de la modalité rhétorique ou de l'insertion syntaxique ont des «comportements» lexicométriques assez contrastés.

- Les litotes en hep + nom à simple négation

29 litotes sont formées de la préposition hep suivie d'un nom axiologiquement neutre. Leurs soixante-trois occurrences leur confèrent un taux de récurrence moyen $(2,17)$. La mieux représentée est hep muy 'seulement', présente à seize exemplaires.

- Les litotes en hep + nom à double négation

Les litotes formées de la préposition hep suivie d'un nom axiologiquement négatif, au nombre de 60 , sont deux fois plus nombreuses que les précédentes. Leurs 219 occurrences leur confèrent un taux de récurrence très supérieur : 3,65 . Cette série n'est pas loin de fournir la moitié du total des litotes relevées dans les cinq textes : $40 \%$ à elle seule. Les plus récurrentes d'entre elles sont hep sy $(50$ occ.) déjà citée, hep mar 'sans doute' (30 occ.) et hep gou 'sans mensonge' (22 occ.).

- Les litotes en hep + bezaf (ou bout) + attribut

Cette série contient un tout petit nombre de litotes (7), toutes représentées une seule fois, à une exception près qui apparaît deux fois : hep bout frealset, hep bezaffrealset. Son taux de récurrence est évidemment tout proche de 1 .

\section{- Les litotes en hep + verbe-nom (+ COD)}

Trente-cinq litotes débutant par hep sont suivies d'un verbenom (autre que bezaf ou bout), éventuellement complété par un COD. On les rencontre soixante dix-neuf fois au fil des vers. Les plus courantes sont hep arretaf'sans hésiter', 'sans perdre de temps' selon le contexte (13 occ.), hep dale 'sans tarder' (9 occ.), hep fellell 'sans manquer' ( 8 occ.) et hep tardaf 'sans tarder' ( 7 occ.). Leur taux de récurrence moyen est de 2,26.

Ces quatre séries en hep additionnées représentent plus des deux 
tiers de l'ensemble des litotes du corpus $(67,15 \%)$. Nombre de leurs ressortissants étaient tellement intégrés à la langue de la prédication (voire à la langue courante) qu'ils n'étaient probablement pas plus sentis comme des litotes que leurs équivalents français d'aujourd'hui 'sans doute' ou 'sans arrêt'.

- Les litotes en $n e+[B E Z-]+$ attribut

Quinze litotes dont le taux de récurrence est légèrement supérieur à 2 (31 occ.) s'ouvrent par une négation suivie de la copule [BEZ-]. Elles apparaissent souvent comme de simples variantes de celles qui sont formées de hep + nom. Ainsi nedeu sy ( 8 occ.), nedeu gou (4 occ.), nedeux mar (2 occ.) sont-elles les équivalents exacts de hep sy, hep gou, hep mar 'assurément'.

- Les litotes en $n e+$ verbe (+COD)

Presque aussi récurrentes que les litotes du 5.1.2. (taux de récurrence 3,47), les 17 litotes formées d'un verbe (autre que [BEZ-]) à la forme négative éventuellement suivi d'un COD apparaissent 59 fois dans le corpus. Elles aussi se présentent parfois comme des formes un peu plus développées que les litotes en hep + nom : hep sy=nedeu $s y=$ ne graf sy; hep dout $=$ nedeux dout $=$ ne graf dout 'assurément'. Cette plasticité morphologico-syntaxique permettait sans doute d'éviter la répétition de formules trop semblables.

- Les litotes phrases

Ces 90 litotes développées en phrases ou en propositions relatives ne se répètent jamais, parce qu'elles ne constituent pas des séries de formules plus ou moins stéréotypées. Intégrées au flux narratif ou à la parole des personnages, elles sont aussi les plus rhétoriques de toutes : si le Christ a résolu de se sacrifier, ne deu eguyt vnan na dou, hoguen tribut an holl tudou 'ce n'est pas pour une ou deux personnes, mais pour payer la rançon de toute l'humanité' (Passion 1410-1411).

\section{La distribution entre textes}

Les litotes sont loin d'être réparties également entre les cinq textes. La Passion, qui représente $63 \%$ du volume, ne contient que 
$53 \%$ du répertoire général des litotes (une pour douze vers); tandis que le Tremenuan, qui n'en représente que $12,5 \%$, contient plus de $18 \%$ de ce répertoire (une pour sept ou huit vers). La Résurrection et Buhez Mabden sont à peu près au même niveau que la Passion (une litote pour douze vers), tandis que les Pemzec Leuenez se situent à un niveau intermédiaire (une litote pour dix vers).

Deux litotes seulement figurent dans les cinq textes : hep sy (50 occ.) et hep mar (30 occ.). Il est vrai que ce sont également les plus récurrentes de toutes, les deux seules que l'on peut à l'occasion soupçonner d'avoir une fonction de «remplissage» du vers. Elles sont avec quelques autres (hep gou, hep dout etc.), me semblet-il, comme les échos intertextuels des «en vérité» qui reviennent si souvent dans les évangiles.

À l'inverse 114 litotes ne figurent que dans un seul des cinq textes. La plupart d'entre elles (105) sont des exemplaires uniques, mais quatre d'entre elles apparaissent deux fois, deux apparaissent trois fois, une quatre fois et une cinq fois.

Enfin la série formelle hep bezaf (ou bout) + attribut tout entière, vingt-trois des cinquante hep + verbe-nom, neuf des vingt-sept $n e+$ verbe $(+C O D)$ et d'autres encore disséminées dans toutes les séries ne sont présentes que dans les Passion et Résurrection. Ce qui pourrait constituer un indice confirmant que les deux textes ont été écrits par le même auteur. En revanche l'auteur du Tremenuan est le seul qui utilise d'assez nombreuses litotes originales comme hep abaff (4 occ.), hep gront (3 occ.), hep trig (3 occ.) etc., ce qui me fait penser qu'il n'a composé aucun des quatre autres textes. De même l'auteur de Buhez Mabden semble-t-il «signer» son œuvre par plusieurs litotes qui n'appartiennent qu'à lui : hep crethat 'imprudemment' (1 occ.), hep sezlou quen 'diligemment' (1 occ.), nedynt fablou 'en vérité' (1 occ.), nedeux goat 'assurément' (1 occ.) etc. Les Pemzec Leuenez sont, elles, trop brèves et contiennent trop peu de litotes pour faire apparaître de telles marques d'auteur.

- La Passion

La Passion (3482 vers) contient 163 litotes différentes totalisant 289 occurrences. 68 d'entre elles ne figurent dans aucun des quatre autres textes. Toutes les séries formelles sont représentées. 
- La Résurrection

La Résurrection (1355 vers) contient 83 litotes différentes totalisant 113 occurrences. Toutes les séries sont représentées.

- Tremenuan an ytron guerchez Maria

Le Tremenuan (764 vers) contient 43 litotes différentes, totalisant 101 occurrences, presque autant que dans la Résurrection qui est presque deux fois plus longue. Cette disproportion, due pour une bonne part à la présence de 27 hep sy et de 12 hep mar 'assurément' (la moitié de tous ceux qui figurent dans l'ensemble du volume) fait de l'auteur de ce texte notre champion de la litote; on peut dire de lui qu'il en use souvent et en abuse parfois. Toutes les séries sont représentées, sauf hep + bezaf + attribut.

\section{- Pemzec Leuenez Maria}

Les Pemzec Leuenez (144 vers) contiennent 13 litotes différentes totalisant 15 occurrences. Toutes les séries sont représentées, sauf hep + bezaf + attribut et ne $+[B E Z-]+$ attribut. Leur auteur n'utilise donc jamais la copule pour former ses litotes. Cela pourrait être également comme une signature (probablement inconsciente), mais en quelque sorte par défaut.

\section{- Buhez Mabden}

Buhez Mabden (359 vers) contient 27 litotes différentes totalisant 30 occurrences. Toutes les séries formelles sont représentées, sauf hep + bezaf + attribut.

\section{Rhétorique et stylistique de la litote}

La multiplicité des formes sémantiquement équivalentes de la litote : Hep sy = hep quet sy = hep quet a sy = hep lacat $s y=$ nedeux $s y=$ nendeu quet $s y=$ ne graf $s y=$ oar nep $s y=$ hep mar na sy 'assurément' fait apparaître une sorte de continuum, de l'expression la plus élémentaire hep + nom à la phrase complète ne allhe ez ve quen 'il ne saurait en être autrement'.

Ainsi se dégagent deux pôles, celui qui porte les formules lapidaires et celui qui produit les formulations syntaxiquement le plus 
développées; entre les deux toute une gradation d'expressions intermédiaires. J'ai évidemment choisi à dessein l'exemple de la litote la plus récurrente et la plus variable du corpus pour illustrer mon propos. Chaque litote répertoriée ne présente pas de réalisations de toutes ses capacités de variabilité, mais toutes sont théoriquement possibles. On peut imaginer à partir de ce seul répertoire l'immense réservoir de litotes potentielles dans lequel les auteurs du temps pouvaient puiser à leur guise, choisissant celle qui convenait le mieux à leur intention présente et au contexte considéré.

J'ai essayé de montrer dans la quatrième partie quels étaient les contextes (une dizaine) les plus favorables à l'apparition de litotes. La question des intentions de leurs auteurs est plus complexe.

\section{La rhétorique}

Toutes les litotes produisent un effet de surlignage : insistant sur la véracité de ce qui est énoncé, sur son intensité, sur son urgence.

Les litotes à négation simple expriment avec une emphase tragique ou ironique selon le cas davantage que ce qu'elles semblent dire si on les entend littéralement : Neonn en bet man pe en manyer an seurt anquen ez soutenher (Passion 660-661) 'Je ne sais vraiment pas comment un tel chagrin sera supportable'; Ne dougiaf pez hoz holl drouguyez vn guez dour (Résurrection 3409-3410) 'toute votre méchanceté ne me fait pas plus peur qu'une goutte d'eau' ou encore Eat ouch hoz hent; en pourentez ne telet dram fez vn guez guyn (Résurrection 4109-4110) que j'ai traduit 'Vous n'avez pas trop mal réussi; en fait de pauvreté on peut, ma foi, trouver pire que vous', et que je comprends littéralement: 'Vous avez fait votre chemin; en tant que pauvre vous ne valez pas, par ma foi, un coup de vin'. Ces trois expressions parmi d'autres nous disent respectivement en réalité : 'je sais très bien qu'il est impossible de supporter une telle douleur', 'je suis parfaitement assuré d'être hors d'atteinte de vos attaques' et 'ne vous plaignez pas, vous êtes un commerçant aisé'. Elles apparaissent dans les dialogues avec un tel naturel qu'une lecture attentive et répétée est nécessaire pour les identifier, surtout si elles sont de forme lapidaire comme celles en hep + nom qui passent fugitivement, comme de simples qualificatifs dans le fil d'un discours. 
Hep est la négation du nom; nendeu (avec ses variantes temporelles et modales) est la négation du qualificatif; ne et $n a$ sont les négations du verbe. Tous ces éléments négatifs sont souvent unis par la syntaxe à d'autres éléments (noms, qualificatifs et verbes) qui contiennent eux-mêmes l'idée d'une négation, d'un manque ou d'un défaut. Ces litotes, que j'ai nommées ci-dessus «à négation double» (3.2.) présentent une construction rhétorique particulièrement intéressante, relevant de l'expression littéraire la plus élaborée.

En français haïr, c'est 'ne pas aimer'; ne pas haïr, c'est donc 'aimer'; Je ne te hais point signifie par conséquent 'je t'aime'. En breton nach 'nier', c'est 'ne pas reconnaître'; ne pas nier, c'est donc 'reconnaître'; nen nachaff quet (Passion 674) 'je ne le nie point' signifierait par conséquent 'je le reconnais, je l'avoue'.

Mais à ce stade on voit bien que la charge sémantique de la litote n'est pas épuisée. L'énergie produite par la "cassure» de la double négation projette rhétoriquement la litote bien au-delà de sa simple résolution arithmétique - «moins par moins égale plus», produisant paradoxalement un effet supplémentaire d'emphase. Lorsque Chimène dit au Cid qu'elle devrait haïr le meurtrier de son père, mais qu'elle ne le fait pas, elle avoue en réalité qu'elle l'aime à la passion. De même lorsque Marie-Madeleine hésite longuement sur le parti à prendre pour retrouver le Christ et qu'elle finit par conclure : me goar perif ne fillif quet (Résurrection 3845) 'je sais ce que je vais faire, je n'y manquerai point', elle affirme en réalité que sa décision est définitive et irrévocable.

Le rôle que jouent ces litotes dans l'élaboration du sens d'une phrase quelconque ne peut ainsi se comprendre que dans le triple saut qu'opère l'esprit pour les intégrer dans leur contexte.

1. Le sens littéral : hep sy 'sans défaut', hep bourt 'sans blague', nen deu queffrin 'ce n'est pas secret', nen nachaff quet 'je ne le nie pas' etc.

2. Le renversement du sens littéral, c'est-à-dire la transformation de la double négation en affirmation : hep sy 'assurément', hep bourt 'c'est vrai', nendeu queffrin 'c'est bien connu', nen nachaff quet 'je l'affirme'.

3. L'amplification rhétorique de l'affirmation: hep sy 'nul ne saurait en douter', hep bourt 'c'est indéniable', nendeu queffrin 'personne ne l'ignore', nen nachaff quet 'on ne me fera pas dire le 
contraire', dans laquelle on retrouve la double négation, dotée cette fois d'une valeur absolue.

\section{La stylistique}

Nous avons jusqu'à présent examiné les litotes du répertoire comme autant d'unités isolées. Replacées dans leur contexte syntaxique, elles apparaissent souvent associées à des formules synonymes, soit par redondance :

Hep poan nac anes nac esmae 'sans souci, ni trouble, ni crainte' = 'dans la quiétude la plus parfaite' (Passion 77);

Pas an drase ne falhe ez ve quet dissimulet na nachet 'cela ne devrait en aucun cas être ni celé ni nié' = 'cela doit être su de tous' (Passion 135) ;

Hep vtilite na mecher 'sans utilité ni besoin' = 'gratuitement' (Passion 237) ;

Hep chenchaff nac exceptaff den 'sans omettre ni excepter quiconque' = 'universellement' (Passion 1354);

Hep mar na gou 'sans doute ni mensonge' = 'incontestablement' (Pemzec Leuenez 5607),

soit par effet de miroir avec une formulation qui exprime la même idée sans revêtir la forme de la litote :

Chetu y glan $\Leftrightarrow$ ne fell vnan 'les voici tous $\Leftrightarrow$ il n'en manque pas un' (Passion 1458);

Ne deuz mar en bet $\Leftrightarrow$ credet plen 'cela ne fait aucun doute $\Leftrightarrow$ croyez-moi bien' (Passion 1659);

noaz pill $\Leftrightarrow$ hep barr dillat 'tout nu $\Leftrightarrow$ sans le moindre vêtement' (Passion 2775);

Cofes a gris $\Leftrightarrow$ ne nachis quet 'J'ai avoué $\Leftrightarrow$ je n'ai pas nié' (Passion 1865);

Ez chomif $\Leftrightarrow$ na ne flachif quet 'Je demeurerai $\Leftrightarrow$ et ne bougerai pas' (Résurrection 3895);

Ezuihet egal haualet $\Leftrightarrow$ hep differancc a tra en bet 'vous deviendrez exactement semblables $\Leftrightarrow$ sans la moindre différence' (Buhez Mabden 5803-5804). 


\section{Synthèse}

La litote n'est que l'un des nombreux tropes qu'en bons lecteurs de la poésie et de la prose latines classiques nos auteurs bretons des quinzième et seizième siècles utilisent couramment : comparaison, métaphore, allégorie, personnification, apostrophe, fausse question, question oratoire, fausse question interro-négative, interjection, redondance du pronom, métonymie, synecdoque, métalepse, ironie, périphrase, hyperbole, accumulation, euphémisme, gradation, hyperbate, redondance, tautologie et peut-être d'autres encore que je n'ai pas identifiés. Elle tient une place de choix dans un arsenal rhétorique dont l'abondance et la précision suffisent à montrer que ses utilisateurs ne sauraient en aucun cas être de médiocres tâcherons du mètre, comme on l'a longtemps cru et répété. Ce n'est que dans le cadre de cette culture rhétorique incontestable que nous pouvons essayer de comprendre le rôle particulier qui est dévolu par eux à la litote.

La litote oblige l'expression à faire un détour : elle nie pour mieux affirmer, elle dit le moins pour faire entendre le plus. L'extrême plasticité de sa morphologie et de sa syntaxe lui permet le plus souvent d'éviter la lourdeur et la monotonie du stéréotype. Ses deux modalités d'énonciation, la négation simple et la négation double, lui permettent de doser l'impact de son irruption dans le discours. L'étendue de ses champs d'application lui permet de placer ses «boucles» négatives à peu près partout où le besoin s'en fait sentir. Enfin, de la même façon qu'une main de Botticcelli se reconnaît au premier coup d'oeil, qu'une phrase musicale de Monteverdi s'identifie dès la première mesure, certaines d'entre elles semblent jouir de la préférence de tel ou tel auteur, faire figure de marques de son «style» personnel.

Ainsi que nous l'avions vu en introduction à propos du français, tous les niveaux de langue du breton peuvent produire des litotes, du plus familier : nedeu bourt 'c'est pas des blagues' (un peu surprenant dans la bouche du Christ) au plus distingué : nedeux queuzet nemedouf 'je suis le plus malheureux des hommes'.

Les litotes ont un dernier caractère un peu troublant : l'ambiguité de leur destinataire. Nous avons vu qu'un très grand nombre d'entre elles, particulièrement les plus récurrentes, attirent l'attention sur la vérité de ce qui est dit (hep sy, hep mar, hep dout...), sur la nécessité 
(hep fellell, hep debataf, hep declinaff...) et sur l'urgence (hep dale, hep arretaf, hep tardaff...) de ce qui est à faire, et aussi sur l'intensité des sentiments (ne songiaff quen, nep no car quet, nez priso den...). $\mathrm{Au}$ sein d'un corpus très majoritairement dramatique, chacune d'entre elles est adressée par un personnage à un autre personnage dans un contexte particulier qui lui donne son sens ${ }^{6}$. Mais si on les prend dans leur masse, elles forment ce que les musiciens nomment un ostinato $^{7}$ et leur fonction conative, considérée de ce point de vue, devient évidente. Indirectement adressées par l'auteur au lecteur ou au spectateur, elles martèlent sans relâche, dans le filigrane des dialogues entre personnages, les vertus exigées du bon chrétien, celles-là même qu'exposent séparément ces personnages : la croyance en la vérité absolue des articles de la foi, la nécessité et l'urgence de se préparer au trépas et au jugement, le zèle dans l'observance des règles morales et sociales. Elles jouent par conséquent un rôle pastoral discret, en quelque sorte «subliminal».

À la différence des véritables chevilles, dont je ne cherche pas à nier l'existence dans cette littérature, chaque litote possède une valeur sémantique particulière, même s'il est vrai que selon le contexte cette valeur est susceptible de varier quelque peu, et même si à l'occasion l'usage de la litote est assurément commode pour le versificateur obligé de suivre des règles prosodiques extrêmement contraignantes.

Dans la foule des tropes utilisés dans la Passion et les quatre autres textes qui lui furent associés en 1530, la litote se distingue ainsi par la force de son appel à la conscience du sens et par l'élégance des multiples détours de son expression.

6. Seules celles qui figurent dans les interventions du héraut, dans le cadre de la prière (Pemzec Leuenez) ou dans celui de la quasi-prédication (Buhez Mabden) s'adressent directement à l'auditoire.

7. «Formule rythmique, mélodique ou harmonique accompagnant de manière immuable les différents éléments thématiques durant tout le morceau» (Wikipedia, ostinato). Les exemples le plus fréquemment cités d'ostinato sont le Canon de Johann Pachelbel et le Boléro de Maurice Ravel. 
